\title{
Construction of targeted insertion mutations in Francisella tularensis subsp. novicida
}

\author{
Jirong Liu, Xhavit Zogaj, Jeffrey R. Barker, and Karl E. Klose \\ University of Texas San Antonio, San Antonio, TX, USA
}

BioTechniques 43:487-492 (October 2007)

doi $10.2144 / 000112574$

Francisella tularensis is one of the most deadly bacterial agents, yet most of the genetic determinants of pathogenesis are still unknown. We have developed an efficient targeted mutagenesis strategy in the model organism F. tularensis subsp. novicida by utilizing universal priming of optimized antibiotic resistance cassettes and splicing by overlap extension (SOE). This process enables fast and efficient construction of targeted insertion mutations in F. tularensis subsp. novicida that have characteristics of nonpolar mutations; optimized targeted mutagenesis strategies will promote the study of this mysterious bacterium and facilitate vaccine development against tularemia. Moreover, the general strategy of gene disruption by PCR-based antibiotic resistance cassette insertion is broadly applicable to many bacterial species

\section{INTRODUCTION}

Francisella tularensis is considered one of the most deadly bacterial agents by the Centers for Disease Control. Yet very little is known about the genetic basis of $F$. tularensis pathogenesis, primarily because of a historical lack of study of this organism. The development of quick and efficient techniques to genetically manipulate $F$. tularensis is critical for a deeper understanding of virulence mechanism(s), which in turn is necessary for the development of effective vaccines against tularemia. F. tularensis subsp. novicida exhibits low virulence in humans and high virulence in mice, but its virulence mechanism(s) appear virtually identical to those employed by $F$. tularensis subsp. tularensis, which is highly virulent to humans. These $F$. tularensis subspecies are also extremely closely related at the genomic level, and thus $F$. tularensis subsp. novicida has emerged as a model to study the pathogenesis of the subspecies more virulent to humans. Recent advances in genetic manipulation have facilitated mutant $F$. tularensis strain construction, including transposon and insertional antibiotic cassette mutagenesis (1-3). However, these types of techniques tend to result in disruption of the expression of downstream genes within the same operon (i.e., polarity). Also, due to the random nature of transposition, this technique requires significant screening of numerous mutants to obtain the desired targeted mutation. We have optimized an effective way to make targeted mutations in $F$. tularensis subsp. novicida that have characteristics of nonpolar mutations.

\section{MATERIALS AND METHODS}

\section{Bacterial Strains}

Escherichia coli strain DH5 $\alpha$ (4) was used for all cloning experiments, and $F$. tularensis subsp. novicida strain U112 (5) was used for strain construction. The $F$. tularensis subsp. novicida $\Delta i g l C::$ erm $C$ strain has been described previously (3).

\section{Plasmid Construction for Antibiotic Resistance Genes}

In order for the antibiotic resistance genes to be efficiently expressed in $F$. tularensis subsp. novicida, we cloned these genes behind the constitutively expressed $F$. tularensis subsp. novicida FTN1451 promoter (6). The FTN1451 promoter was PCR-amplified with $\mathrm{FpUpBg} / \mathrm{II}$ and $\mathrm{FpDnNdeI}$ primers, digested with $B g l \mathrm{II}$ and $N d e \mathrm{I}$, and ligated into pET15b (Novagen,
Madison, WI, USA) that had been similarly digested to form pKEK886; this construct has the FTN1451 promoter in place of the $\mathrm{T} 7$ promoter. Then, the erythromycin, kanamycin, and chloramphenicol resistance genes (7-9) were amplified with ErmCFNdeI and ErmCRBamHI, KanFNdeI and KanRBamHI, and CATFNdeI and CATRBamHI, respectively. The three fragments were each cut with BamHI and NdeI and inserted into similarly digested pKEK886, resulting in pKEK887 (FpermC), pKEK898 (FpKan), and pKEK923 (Fpcat), respectively.

\section{Primer Design and PCR Conditions}

Universal priming sites were designed from the backbone sequence of pET15b to facilitate amplification of any antibiotic resistance gene inserted between the two priming sites (i.e., in pKEK887, pKEK898, and pKEK923). The universal primers used to PCR-amplify the antibiotic resistance genes are UniUp, 5'-TGCAT TAGGAAGCAGCCCAGTAGT$3^{\prime}$ and UniDn, 5'-TTCCTTTCGG GCTTTGTTAGCAGC-3'. Complementary sequences were incorporated into the primers used to amplify the F. tularensis subsp. novicida flanking DNA regions necessary to construct the gene knockout. As an example, the sequences of the primers used to knock out the iglB gene (FTT1358, FTN1323) are described here. The upstream 904-bp iglB flanking fragment was PCR-amplified with primers IglBUp1, 5'-GAATTCGTCGACGTGTCTTAGC AACTGTACCAGCTAGAGG-3' (SalI site is in bold) and IglBUp2, $5^{\prime}$-ACTAC TGGGCTGCTTCCTAATGCAGCGC CATAAGGTTTCTAGCATTGTAGTC$3^{\prime}$ (note the complementary sequence to UniUp is underlined). The downstream 929-bp iglB flanking fragment was PCRamplified with primers IglBDn1, 5'-GC TGCTAACAAAGCCCGAAAGGAAA TCGAGGTTGAAACCATACCGGGT$3^{\prime}$ (note the complementary sequence to UniDn is underlined) and IglBDn2, $5^{\prime}-\mathrm{G}$ AATTCGTCGACGGCAAATAGCTT GCGGTGCTTAAC-3' (SalI site in bold). Chromosomal DNA from $F$. tularensis subsp. novicida U112 was utilized as the template in PCRs with IglB primers. PCR 

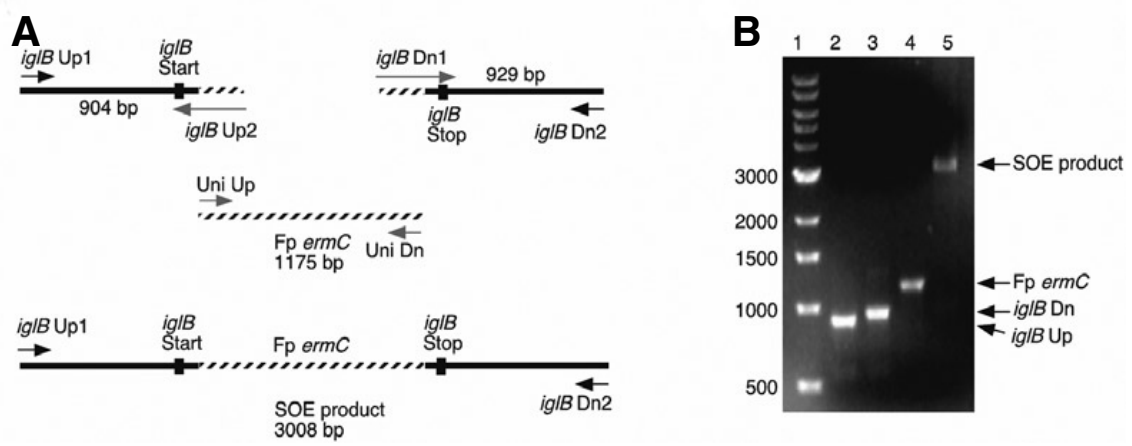

Figure 1. Construction of Francisella tularensis targeted mutations. (A) The splicing by overlap extension (SOE) technique. Universal primers (UniUp and UniDn) are used to PCR-amplify antibiotic resistance cassettes, while specific primers are used to PCR-amplify flanking regions of the gene to be inactivated, resulting in three individual fragments. The $3^{\prime}$ primer of the upstream fragment (iglBUp2) and the $5^{\prime}$ primer of the downstream fragment (iglBDn1) incorporate the universal priming sites. In an SOE-PCR, all three fragments are used as template with the outermost primers (iglBUp1 and iglBDn2). (B) The products of the individual and SOE-PCRs are shown: lane 1, 1-kbp ladder (Gibco BRL, Bethesda, MD, USA); lane 2, iglBUp1 + iglBUp2; lane 3, iglBDn1 + iglBDn2; lane 4, UniUp + UniDn amplifying Fperm C; lane 5, SOE-PCR product.

conditions were $10 \mathrm{ng}$ DNA, $5 \mu \mathrm{M}$ each primer, $8 \mathrm{mM}$ dNTPs, $5 \mu \mathrm{L} 10 \times$ buffer, $0.5 \mu \mathrm{L}$ KOD XL DNA polymerase (Novagen) in $50 \mu \mathrm{L}$ total volume. The PCR program utilized was $94^{\circ} \mathrm{C} 10 \mathrm{~min}$; followed by 30 cycles of $94^{\circ} \mathrm{C} 60 \mathrm{~s}, 60^{\circ} \mathrm{C}$ $80 \mathrm{~s}$, and $72^{\circ} \mathrm{C} 110 \mathrm{~s}$; and a final $72^{\circ} \mathrm{C}$ step for $10 \mathrm{~min}$.

The three individual PCR fragments generated from primer combinations UniUp+UniDn (in this example we utilized the FpermC fragment amplified from pKEK887), IglBUp1+Ig1BUp2 (upstream fragment), and IglBDn1+IglBDn2 (downstream fragment) were separated by agarose gel electrophoresis and purified (Qiagen, Valencia, CA, USA). The template DNA for the splicing by overlap extension (SOE)-PCR was prepared by adding $100 \mathrm{ng}$ of each fragment into a mixture with a total volume of 10 $\mu \mathrm{L}$, then using $1 \mu \mathrm{L}$ of this mixture for the PCR. PCR conditions for the SOE reaction were $1 \mu \mathrm{L}$ threefragment DNA mixture, $5 \mu \mathrm{M}$ each IglBUp 1 and IglBDn 2 primers, $8 \mathrm{mM}$ dNTPs, $5 \mu \mathrm{L} 10 \times$ buffer, $0.5 \mu \mathrm{L}$ KOD XL DNA polymerase (Novagen), $0.5 \mathrm{mM} \mathrm{MgCl}_{2}$, and $1 \%$ dimethyl sulfoxide (DMSO) in a total volume of $50 \mu \mathrm{L}$. The PCR program utilized was identical to that detailed in this section. The resulting fragment corresponding to the correct size of 3008 bp was digested with SalI, then separated by agarose gel electro- phoresis, purified, and ligated with pGEM-5Zf (Promega, Madison, WI, USA) digested similarly to form pKEK1118 ( $\Delta i g l B::$ erm $C)$. The plasmid was sequenced to verify the construct was correct.

\section{Francisella tularensis subsp. novicida Strain Construction}

F. tularensis subsp. novicida strain U112 was grown at $37^{\circ} \mathrm{C}$ into tryptic soy broth plus (TSBP) media $(40 \mathrm{~g} / \mathrm{L}$ tryptic soy broth with $0.1 \%$ cysteine, $25 \% \mu \mathrm{g} / \mathrm{mL}$ ferrous sulfate, $25 \mu \mathrm{g} / \mathrm{mL}$ sodium pyruvate, and $25 \mu \mathrm{g} / \mathrm{mL}$ sodium metasulfite) to an approximate $\mathrm{OD}_{600}$ value of $0.6-0.8$. The culture was then pelleted and washed twice with cold water, then resuspended in cryo-transformation buffer (10\% glycerol, $10 \mathrm{mM}$ HEPES, $\mathrm{pH}$ 6.5, $0.1 \mathrm{M}$ calcium chloride, and $10 \mathrm{mM}$ rubidium chloride). Two to ten micrograms of plasmid pKEK1118 were added to cryo-competent cells, and the mixture was incubated in an ice bath for $30 \mathrm{~min}$. The mixture was then flash-frozen in dry ice-ethanol bath for $5 \mathrm{~min}$, then placed in a room temperature water bath for $5 \mathrm{~min}$, and directly spread onto TSBP-agar plates with appropriate antibiotics (in this example, $100 \mu \mathrm{g} / \mathrm{mL}$ erythromycin). Plates were incubated at $37^{\circ} \mathrm{C}$ for 48-72 h. Transformations typically yielded 100-1000 resistant colonies. Transformation can also be performed via electroporation (10); however the strain described here was constructed using cryo-transformation. PCR was performed on $\mathrm{Erm}^{\mathrm{R}}$ colonies with the primer pairs IglBFor and IglBRev; PCR was performed as described in the section entiled Primer Design and PCR Conditions, with the template prepared by scraping a small amount of colony, resuspending in $100 \mu \mathrm{L}$ water, and performing PCR with iglB primers. The PCR product was subjected to restriction analysis with $B g l \mathrm{II}$ and sequencing to confirm the presence of the $\Delta i g l B::$ erm $C$ mutation. The loss of the plasmid backbone was confirmed with primers specific for the pUC ori (present in pGEM-5Zf; primers pUCoriF and pUCoriR).

\section{Western Immunoblot}

Whole cell extracts of $F$. tularensis subsp. novicida strains were separated by $10 \%$ sodium dodecyl sulfate polyacrylamide gel electrophoresis (SDS-PAGE) and subjected to Western immunoblot utilizing goat polyclonal anti-IglC antibodies; detection was performed with the ECL $^{\mathrm{TM}}$ detection kit (GE Healthcare, Piscataway, NJ, USA).

\section{RESULTS AND DISCUSSION}

We developed a relatively quick and efficient means to inactivate genes in $F$. tularensis subsp. novicida by optimized antibiotic resistance cassettes, optimized priming sites, and SOE-PCR. We constructed optimized antibiotic resistance cassettes by placing the genes for erythromycin, chloramphenicol, and kanamycin resistance under control of a strong constitutive F. tularensis promoter (FTN1451p). Each of these resistance cassettes was flanked by the same universal priming sites, and this facilitated the insertion of any of these resistance markers into the gene of interest.

The gene knockout construct was assembled by SOE-PCR $(11,12)$. Fragments flanking the gene to be knocked out were first PCR-amplified with primers that also incorporate the universal priming sites. A minimum of 500-bp flanking homology was 
Table 1. Oligonucleotides Used in This Study

\begin{tabular}{ll}
\hline Oligonucleotide & Sequence (Restriction Sites are Underlined) \\
FpUpBgllI & 5'-GGGAGATCTTTTGGGTTGTCACTCATCGTATTTG-3' \\
FpDnNdel & 5'-CCCGGGCATATGTTTAGTCTCCTTTTTTTGTTA-3' \\
ErmCFNdel & 5'-GGAATTCCATATGAACGAGAAAAATATAAAAC-3' \\
ErmCRBamHI & 5'-CGCGGATCCTTACTTATTAAATAATTTATAGCT-3' \\
KanFNdel & 5'-GGAATTCCATATGAGCCATATTCAACGGGAA-3' \\
KanRBamHI & 5'-CGCGGATCCTTAGAAAAACTCATCGAGCATCAAATG-3' \\
CatFNdel & 5'-GGAATTCCATATGGAGAAAAAATCACTGGATATACC-3' \\
CatRBamHI & 5'-CGCGGATCCTTTAGTAAGCCGGATCCACGC-3' \\
UniUp & 5'-TGCATTAGGAAGCAGCCCAGTAGT-3' \\
UniDn & 5'-TTCCTTTCGGGCTTTGTTAGCAGC-3' \\
IgIBUp1 & 5'-GAATTCGTCGACGTGTCTTAGCAACTGTACCAGCTAGAGG-3' \\
IgIBUp2 & 5'-ACTACTGGGCTGCTTCCTAATGCAGCGCCATAAGGTTTCTAGCATTGTAGTC-3' \\
IgIBDn1 & 5'-GCTGCTAACAAAGCCCGAAAGGAAATCGAGGTTGAAACCATACCGGGT-3' \\
IgIBDn2 & 5'-GAATTCGTCGACGGCAAATAGCTTGCGGTGCTTAAC-3' \\
IgIBFor & 5'-GTCAGAAGAGTAAATAATGGTGT-3' \\
IgIBRev & 5'-GGCTCTATACTAATACTAAAAGC-3' \\
pUCoriF & 5'-ATGTGAGCAAAAGGCCAGCAA-3' \\
pUCoriR & 5'-CCGTAGAAAAGATCAAAGGATC-3'
\end{tabular}

necessary for homologous recombination in F. tularensis subsp. novicida (3). As an example of this technique, we illustrate here the inactivation of the iglB gene (Figure 1). The PCR fragment upstream of iglB incorporated one of the universal priming sites at its $3^{\prime}$ end, while the PCR fragment downstream of $i g l B$ incorporated the other universal priming site at its $5^{\prime}$ end. These fragments were PCR-amplified separately in the first reaction, and the antibiotic cassette is

also PCR-amplified separately with the universal primers. In a second PCR, all three fragments (iglB upstream, iglB downstream, and antibiotic cassette fragments) were added to the reaction at a ratio of 1:1:1 along with the outermost primers. Frequently, smaller amplified fragments could be visualized in the SOE reaction, but these could be ignored in favor of the fragment of the correct size.

The resulting fragment of the anticipated correct size (in this case

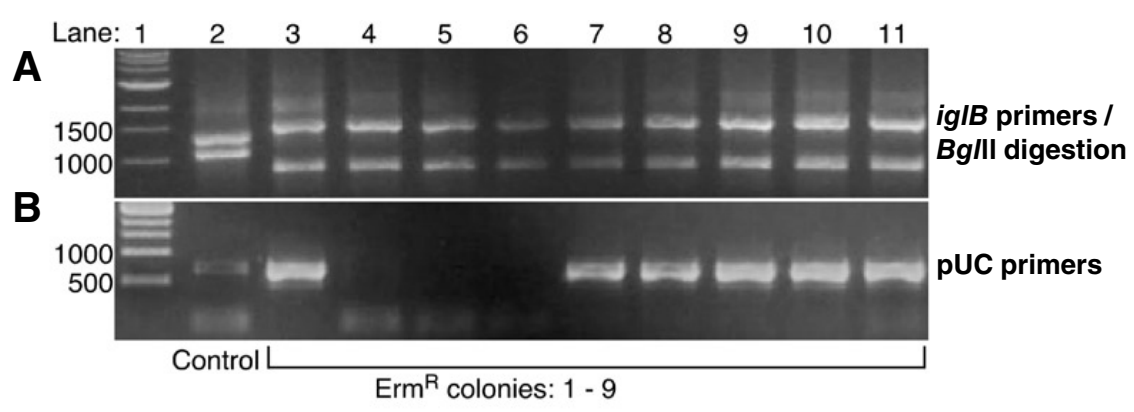

Figure 2. PCR screen of $\mathbf{E r m}^{\mathrm{R}}$ colonies. Francisella tularensis subsp. novicida was transformed with pKEK1118 ( $\triangle$ iglB::ermC) and Erm $^{\mathrm{R}}$ colonies screened by PCR. (A) iglB primers IglBFor + IglBRev used in PCR (amplify 2420-bp fragment in wild-type strain, and 2382-bp fragment in correct construct), followed by $B g l I I$ digestion of resulting fragment. $B g l I I$ site present in antibiotic resistance cassette (in $\mathrm{FpUp} B g l \mathrm{II}$ as described in the Materials and Methods section) allows for diagnostic identification of $\Delta i g l B$ ::erm C construct with $B g l I I$ digestion (1550- and 832-bp fragments); there is a natural $B g l I I$ site within $i g l B$ gene, resulting in two fragments for the wild-type $i g l B$ region of different sizes following $B g l \mathrm{II}$ digestion (1300- and 1120-bp fragments). Lane 1, 1-kbp ladder; lane 2, U112 (wild-type); lanes 3-11, Erm ${ }^{\mathrm{R}}$ colonies 1-9. (B) pUC ori primers pUCoriF + pUCoriR used in PCR (amplify 673-bp pUC ori fragment). Lane 1, 1- kbp ladder; lane 2, pKEK1118; lanes 3-11, Erm ${ }^{\mathrm{R}}$ colonies 1-9.
$3008 \mathrm{bp}$; see Figure 1) was then cloned into a vector; we utilized pGEM-5Zf, but any plasmid vector that fails to replicate in $F$. tularensis will suffice. Alternatively, the purified PCR fragment can be immediately transformed into $F$. tularensis subsp. novicida to achieve a gene knockout by homologous recombination (3), but because of the low yield of full-length product in PCR and the large amount of DNA necessary for transformation, we found it easiest to subclone this fragment into a vector prior to transformation. The low GC content of $F$. tularensis DNA makes the primer design critical for SOE-PCR. We achieved the most consistent results by designing primers to regions with approximately $50 \%$ GC content, and an annealing temperature of at least $60^{\circ} \mathrm{C}$. It is also important to keep the optimal equivalent ratio for the three individual fragments $(1: 1: 1)$ in order to achieve amplification of the fulllength fragment in the SOE-PCR. Occasionally the annealing temperature needed to be reduced to $55^{\circ} \mathrm{C}$ to obtain full-length PCR product.

The full-length knockout construct (e.g., $\Delta i g l B:: e r m C$ ), either cloned into a vector or in the linear PCR fragment, is then transformed into the $F$. tularensis subsp. novicida U112 strain via cryo-transformation (3) or electroporation (10). Transformed cells were then plated on media selective for the antibiotic resistance cassette within the knockout construct (in this case $\operatorname{Erm}^{\mathrm{R}}$ ). Resistant colonies were screened by colony PCR with primers specific for the region being mutated, followed by restriction analysis for a $B g l$ II site (present in FpUpBglII), which was diagnostic for the correct construct. This analysis confirmed that the plasmid had integrated into the chromosome of all nine $\mathrm{Erm}^{\mathrm{R}}$ colonies screened (Figure 2A). Additional PCR analysis included primers for the ori of the vector, to determine whether the plasmid remained integrated in the chromosome (Figure 2B). With the majority of knockout constructs, we found that a second recombination occurred relatively rapidly and the plasmid vector was lost in approximately $30 \%-70 \%$ of colonies screened from the primary transformation plate 


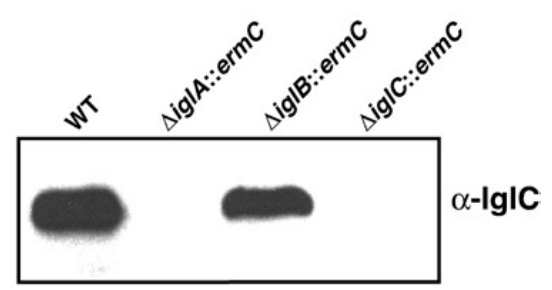

Figure 3. IglC Western immunoblot of Francisella tularensis subsp. novicida mutants. Equivalent amounts of whole cell lysates of wild-type (U112), $\Delta i g l A::$ erm C, $\Delta i g l B::$ erm C, and $\Delta i g l C:: e r m C$ strains were separated by $10 \%$ sodium dodecyl sulfate polyacrylamide gel electrophoresis (SDS-PAGE), and probed with $\alpha \mathrm{IglC}$ by Western immunoblot. $\triangle i g l A:$ :erm $C$ strain was constructed by previously described methods (Reference 3), while $\Delta i g l B:$ :ermC strain was constructed as described here.

(in the example shown, three of nine $\mathrm{Erm}^{\mathrm{R}}$ colonies have already lost the plasmid; Figure 2B, lanes 4-6). As can be seen in the figure, only the $\Delta i g l B:: e r m C$ pattern and not the wildtype pattern could be detected in the Erm $^{\mathrm{R}}$ colonies. With other constructs, additional growth in liquid media followed by plating on selective media and subsequent screening resulted in the identification of the correct mutant colonies. The deletion of the iglB gene in the $\Delta i g l B:$ :erm $C$ mutants was confirmed by additional PCR analysis and sequencing, as well as phenotypic characterization (intramacrophage growth and mouse virulence).

The iglB gene lies within an operon (iglABCD), and disruption of this gene could lead to polar effects reducing or eliminating expression of the downstream genes. A $\Delta$ iglA:: ermC F. tularensis subsp. novicida mutation constructed by previously developed techniques (3) is polar on IglC expression, whereas the $\Delta i g l B:$ : erm $C$ mutation constructed by the techniques described here is not polar on IglC expression, as determined by Western immunoblot (Figure 3 ). Polarity is defined by an alteration of the expression of the downstream genes, thus the Western blot demonstrates that the $\Delta i g l B::$ erm $C$ mutation does not appear to have polar effects on IglC expression. The salient difference between the iglA and iglB mutations is the presence of the $F$. tularensis promoter that drives the expression of ermC in the $\triangle i g l B::$ erm $C$ mutation; this promoter is absent from the $\Delta i g l A:: e r m C$ mutation. Thus, the optimized technique described here to disrupt genes in $F$. tularensis subsp. novicida leads to insertion mutations that have the characteristics of nonpolar mutations, which is important for the study of genes within multigene operons.

The general technique of optimizing antibiotic resistance gene cassettes and utilizing universal primers in optimized SOE PCRs to create gene knockout constructs is easily adapted for usage in many bacterial species, and we have used this technique successfully for gene knockouts in Vibrio cholerae as well. Due to the AT-rich nature of the $F$. tularensis promoter driving antibiotic resistance in the constructs described here, these cassettes are likely to be expressed at high levels in many bacterial species, but the antibiotic cassettes can be optimized for any bacterium by insertion of an appropriate promoter. Many bacterial species cannot efficiently utilize linear DNA for transformation and subsequent double homologous recombination, and thus the assembled knockout construct would need to be cloned into a suicide vector suitable for that particular species. Because the knockout construct is assembled by PCR techniques using universal priming sites that flank multiple antibiotic resistance cassettes, we can easily and quickly construct the same knockout with different antibiotic resistances inserted (e.g., $\Delta i g l B::$ erm $C, \quad \Delta i g l B:: c a t, \quad \Delta i g l B::$ kan) by simply mixing and matching the components during the SOEPCR. Since the assembly of the gene knockout proceeds by PCR techniques rather than subcloning, the technique described here dramatically decreases the time required to construct targeted gene knockouts in F. tularensis subsp. novicida, compared with previously described techniques (3).

\section{ACKNOWLEDGMENTS}

This project has been funded in whole or in part with Federal funds from the National Institute of Allergies and Infectious Diseases, National Institutes of Health, Department of
Health and Human Services, under contract no. HHSN266200500040C.

\section{COMPETING INTERESTS STATEMENT}

The authors declare no competing interests.

\section{REFERENCES}

1. Anthony, L.S., M.Z. Gu, S.C. Cowley, W.W. Leung, and F.E. Nano. 1991. Transformation and allelic replacement in Francisella spp. J. Gen. Microbiol. 137:2697-2703.

2. Kawula, T.H., J.D. Hall, J.R. Fuller, and R.R. Craven. 2004. Use of transposontransposase complexes to create stable insertion mutant strains of Francisella tularensis LVS. Appl. Environ. Microbiol. 70:6901-6904.

3. Lauriano, C.M., J.R. Barker, F.E. Nano, B.P. Arulanandam, and K.E. Klose. 2003. Allelic Exchange in Francisella tularensis using PCR products. FEMS Microbiol. Lett. 229:195-202.

4. Hanahan, D. 1983. Studies on transformation of Escherichia coli with plasmids. J. Mol. Biol. 166:577-580.

5. Anthony, L.D., R.D. Burke, and F.E. Nano. 1991. Growth of Francisella spp. in rodent macrophages. Infect. Immun. 59:3291-3296.

6. Gallagher, L.A., E. Ramage, M.A. Jacobs, R. Kaul, M. Brittnacher, and C. Manoil. 2007. A comprehensive transposon mutant library of Francisella novicida, a bioweapon surrogate. Proc. Natl. Acad. Sci. USA 104:1009-1014.

7. Monod, M., C. Denoya, and D. Dubnau. 1986. Sequence and properties of pIM13, a macrolide-lincosamide-streptogramin B resistance plasmid from Bacillus subtilis. J. Bacteriol. 167:138-147.

8. Rose, R.E. 1988. The nucleotide sequence of pACYC177. Nucleic Acids Res. 16:356.

9. Rose, R.E. 1988. The nucleotide sequence of pACYC184. Nucleic Acids Res. 16:355.

10.Baron, G.S., S.V. Myltseva, and F.E. Nano. 1995. Electroporation of Francisella tularensis, pp. 149-154. In J.A. Nickoloff (Ed.), Meth. Mol. Biol. Humana Press, Inc., Totowa, NJ.

11.Ho, S.N., H.D. Hunt, R.M. Horton, J.K. Pullen, and L.R. Pease. 1989. Site-directed mutagenesis by overlap extension using the polymerase chain reaction. Gene 77:51-59.

12.Horton, R.M., H.D. Hunt, S.N. Ho, J.K. Pullen, and L.R. Pease. 1989. Engineering hybrid genes without the use of restriction enzymes: gene splicing by overlap extension. Gene 77:61-68.

Received 11 June 2007; accepted 18 July 2007. 
Address correspondence to Karl E. Klose,

Department of Biology, University of Texas San Antonio, One UTSA Circle, San Antonio, TX 78249, USA. e-mail: kklose@utsa.edu

To purchase reprints of this article, contact: Reprints@BioTechniques.com 